\title{
Accuracy and impact of Xpert MTB/RIF for the diagnosis of smear-negative or sputum-scarce tuberculosis using bronchoalveolar lavage fluid
}

\author{
Grant Theron, ${ }^{1}$ Jonny Peter, ${ }^{1}$ Richard Meldau, ${ }^{1}$ Hoosain Khalfey, ${ }_{1}$ Phindile Gina, ${ }^{1}$ \\ Brian Matinyena, ${ }^{1}$ Laura Lenders, ${ }^{1}$ Gregory Calligaro, ${ }^{1}$ Brian Allwood, ${ }^{1}$ \\ Gregory Symons, ${ }^{1}$ Ureshnie Govender, ${ }^{1}$ Mashiko Setshedi, ${ }^{1}$ Keertan Dheda ${ }^{1,2}$
}

\begin{abstract}
- Additional material is published online only. To view please visit the journal online (http://dx.doi.org/10.1136/ thoraxjnl-2013-203485).

${ }^{1}$ Department of Medicine, Lung Infection and Immunity Unit, Division of Pulmonology \& UCT Lung Institute, University of Cape Town, Cape Town, Western Cape, South Africa

${ }^{2}$ Institute of Infectious Diseases and Molecular Medicine, University of Cape Town, Cape Town, Western Cape, South Africa
\end{abstract}

\section{Correspondence to} Dr Keertan Dheda, Department of Medicine, Lung Infection and Immunity Unit, University of Cape Town, H47 Old Main Building, Groote Schuur Hospital, Observatory, Cape Town, Western Cape 7925.

South Africa;

keertan.dheda@uct.ac.za

Received 4 March 2013 Revised 27 May 2013 Accepted 4 June 2013 Published Online First 29 June 2013

\section{ABSTRACT}

Rationale The accuracy and impact of new tuberculosis (TB) tests, such as Xpert MTB/RIF, when performed on bronchoalveolar lavage fluid (BALF) obtained from patients with sputum-scarce or smear-negative TB is unclear.

Methods South African patients with suspected pulmonary TB $(n=160)$ who were sputum-scarce or smear-negative underwent bronchoscopy. MTB/RIF was performed on uncentrifuged BALF (1 ml) and/or a resuspended pellet of centrifuged BALF $(\sim 10 \mathrm{ml})$. Time to TB detection and anti-TB treatment initiation were compared between phase one, when MTB/RIF was performed as a research tool, and phase two, when it was used for patient management.

Results 27 of 154 patients with complete data had culture-confirmed TB. Of these, a significantly lower proportion were detected by smear microscopy compared with MTB/RIF (58\%, $95 \% \mathrm{Cl} 39 \%$ to $75 \%$ versus $93 \%$, $77 \%$ to $98 \% ; p<0.001)$. Of the 127 patients who were culture negative, $96 \%$ (91\% to $98 \%$ ) were MTB/RIF negative. When phase two was compared with phase one, MTB/RIF reduced the median days to TB detection $(29(18-41)$ to $0(0-0) ; p<0.001)$. However, more patients initiated empirical therapy (absence of a positive test in those commencing treatment) in phase one versus phase two (79\% (11/14) versus 28\% (10/25); $\mathrm{p}=0.026)$. Consequently, there was no detectable difference in the overall proportion of patients initiating treatment $(26 \%(17 / 67 ; 17 \%$ to $37 \%)$ versus $36 \%$ $(26 / 73 ; 26 \%$ to $47 \%) ; p=0.196)$ or the days to treatment initiation (10 (1-49) versus $7(0-21)$; $\mathrm{p}=0.330$ ). BALF centrifugation, HIV coinfection and a second MTB/RIF did not result in detectable changes in accuracy.

Conclusions MTB/RIF detected TB cases more accurately and more rapidly than smear microscopy and significantly reduced the rate of empirical treatment.

\section{INTRODUCTION}

Tuberculosis (TB) is the leading cause of morbidity and mortality in patients with HIV infection in sub-Saharan Africa. ${ }^{1}$ Sputum-based smear microscopy is commonly used to diagnose TB, however its sensitivity is poor $(40-60 \%) .^{2}$ In South Africa, patients who are smear negative or sputum scarce represent up to a third of those with pulmonary $\mathrm{TB}^{3},{ }^{34}$ and these individuals are often at an increased risk of poor

\section{Key messages}

What is the key question?

- What is the accuracy of Xpert MTB/RIF for the detection of tuberculosis (TB) when performed on bronchoalveolar lavage fluid, and how does the test impact anti-TB treatment initiation?

What is the bottom line?

- Xpert MTB/RIF detected almost all microbiological confirmed cases of TB and resulted in same day diagnosis.

\section{Why read on?}

- Xpert MTB/RIF reduced the proportion of patients who initiated treatment in the absence of a positive test result; however, Xpert MTB/ RIF did not increase the overall proportion of patients initiating treatment due to high rates of empirical anti-TB treatment in the phase before the test was used to guide patient management.

outcomes due to the associated delay in making a diagnosis. $^{5}$

Obtaining a high-quality biological specimen for testing is key to reducing the diagnostic delay. Although sputum induction is frequently used, it fails to provide a specimen of adequate volume or quality in up to $20 \%$ of cases ${ }^{6}$ and does not enable the airways to be visualised or biopsied. Thus, bronchoscopy with bronchoalveolar lavage or bronchial washings is often performed, when available, in patients who are smear negative or sputum scarce.

Newer nucleic acid amplification technologies, such as the World Health Organization (WHO)endorsed Xpert MTB/RIF assay (Cepheid, USA), represent a significant advance over smear microscopy. ${ }^{8}$ However, MTB/RIF still does not detect significant proportions of patients who are smear negative or infected with HIV (sensitivities of $68 \%$ and $80 \%$, respectively). ${ }^{9}$

Although the accuracy of MTB/RIF when performed on sputum is well validated, ${ }^{10}$ little is known about its performance in bronchoalveolar 
lavage fluid (BALF). There are limited data from a few studies evaluating a small number of specimens, ${ }^{11}{ }^{12}$ and none from a setting with high HIV prevalence. Furthermore, there is no information regarding whether MTB/RIF testing of BALF can improve the proportion of patients initiating treatment or the time-to-treatment initiation in settings where rates of empirical treatment are high.

To better understand these issues, we prospectively examined the accuracy of MTB/RIF performed on BALF obtained from smear-negative or sputum-scarce patients with suspected pulmonary TB recruited from a hospital in Cape Town, South Africa. We assessed the impact of MTB/RIF on time to diagnosis, the proportion of patients who initiated treatment (overall and empirically), and the time to treatment initiation before (phase one) and after it was used for patient management (phase two).

\section{MATERIALS AND METHODS}

\section{Study sites and population}

We prospectively enrolled patients ( $\geq 18$ years of age) with suspected pulmonary TB who were referred for bronchoscopy to the respiratory clinic at Groote Schuur Hospital in Cape Town, South Africa ( $n=160$; figure 1) between December 2007 and November 2012. Patients had at least one symptom of TB, a chest radiograph with infiltrates compatible with active $\mathrm{TB}$, at least two consecutive sputum smear microscopy results negative for acid-fast bacilli (within 2 weeks of enrolment) or were unable to self-expectorate sputum. A bronchoscopy also had to be judged to be safe and feasible. MTB/RIF results were, prior to the WHO endorsement of the assay for sputum testing in February $2011,{ }^{7}$ not reported to clinical staff and not used for patient management (phase one). Following its endorsement, MTB/RIF was used to guide the initiation of anti-TB treatment (phase two). Aside from the use of MTB/RIF for patient management in phase two, the study conditions, eligibility criteria, and clinical staff were the same in both phases.

\section{Patient evaluation and specimen collection}

Detailed patient and laboratory-specific information was recorded on a standardised case record form. An HIV test was performed after appropriate counselling. Bronchoscopy was performed by clinical investigators (KD, HK, GS, GC, BA) by placement of the bronchoscope into an airway of an affected lung segment, and the instillation of sterile saline $(0.9 \%)$, usually to a volume of 60-120 ml. Bronchial brushings and/or a transbronchial biopsy was obtained if technically feasible and appropriate for the clinical investigation of that patient.

\section{Routine diagnostic tests}

BALF was split and one arbitrarily selected aliquot sent immediately to a reference laboratory attached to Groote Schuur Hospital for NALC-NaOH decontamination. The second aliquot was used for MTB/RIF testing. The decontaminated specimen was examined twice by concentrated fluorescence smear microscopy and underwent liquid culture for Mycobacterium tuberculosis using the BACTEC MGIT 960 system (BD Diagnostics, USA). ${ }^{13}$ Culture-positive isolates underwent routine phenotypic drug susceptibility testing for rifampicin and isoniazid using the MGIT 960 SIRE kit ${ }^{14}$ from December 2008. When requested, concentrated fluorescence smear microscopy and liquid culture were also performed on bronchial brushings and transbronchial biopsy specimens. Histological examination was undertaken on transbronchial biopsies, when available.

\section{Patient classification}

Patients were classified as definite $\mathrm{TB}$ if they had at least one specimen culture positive for $\mathrm{TB}$ or, upon histological investigation of the transbronchial biopsy, were reported to contain caseating or necrotising granulomatous inflammation compatible with TB. Patients who were BALF culture negative for TB and had no histological evidence of active TB disease were classified either as possible TB (if initiated on anti-TB treatment based on clinical suspicion) or as non-TB (if not initiated on anti-TB treatment). A patient was empirically treated if treatment was initiated by the attending physician in the absence of a positive test result (further details on empirical treatment initiation is given in the online supplementary material).

\section{Xpert MTB/RIF and patient management}

An aliquot of BALF was either frozen at $-20^{\circ} \mathrm{C}$ for later batched testing in phase one, or used immediately for MTB/RIF testing in phase two. Thus, in phase two, MTB/RIF was used to guide patient management whereas in phase one it was performed for research use only, and treatment was guided by smear microscopy and culture. The assay was performed on $1 \mathrm{ml}$ of uncentrifuged BALF and, when available, a median volume (IQR) of $10 \mathrm{ml}$ (5$10)$ of BALF centrifuged $(3000 \times \mathrm{g}, 15 \mathrm{~min})$ at room temperature and, following decantation of the supernatant, resuspended in $1 \mathrm{ml}$ of sterile phosphate-buffered saline. The MTB/RIF procedure was then followed as described. ${ }^{15} 16$ The laboratory technician performing the procedure was blinded to patient information and test results. MTB/RIF results in phase two were reported immediately to clinical staff.

\section{Analysis of discordant Xpert MTB/RIF and culture results}

When sufficient BALF remained, a Genotype MTBDRplus assay (version two; Hain Lifesciences) ${ }^{17}$ was performed on specimens that where either MTB/RIF positive but culture and histology negative for active TB; or specimens that were MTB/RIF positive for $M$ tuberculosis complex and rifampicin resistant, but had phenotypically susceptible culture isolates.

\section{Statistical analysis}

We followed the STARD criteria. ${ }^{18}$ For the primary analysis of MTB/RIF sensitivity and specificity on BALF, culture positivity from the same specimen served as a reference standard. A secondary analysis was performed versus culture positivity from any specimen and/or a histological profile compatible for active TB. A third analysis was also performed when the possible TB group was included in the definite TB group. Statistical analyses were performed using OpenEpi (V.2.3.1) ${ }^{19}$ and Graphpad Prism (V.5.0; GraphPad Software, USA, http://www.graphpad.com). We used the D'Agostino-Pearson omnibus test to detect for normality. The Mann-Whitney test was used to compare nonparametric median time to detection or treatment. The Mid-P exact test was used for comparisons between proportions. CIs were calculated using the Wilson Score method.

\section{RESULTS}

\section{Study population and TB diagnoses}

Of the 160 patients with suspected pulmonary TB who were referred for bronchoscopy and consented to participate in the study, six were excluded from the analysis as indicated in figure 1. Of the 154 included patients, 111 (72\%) were sputum scarce and $46(35 \%)$ were HIV infected (table 1). Twenty-seven of the 154 patients (18\%) were BALF culture positive for $M$ tuberculosis. When a bronchial brushing or transbronchial biopsy was cultured, 


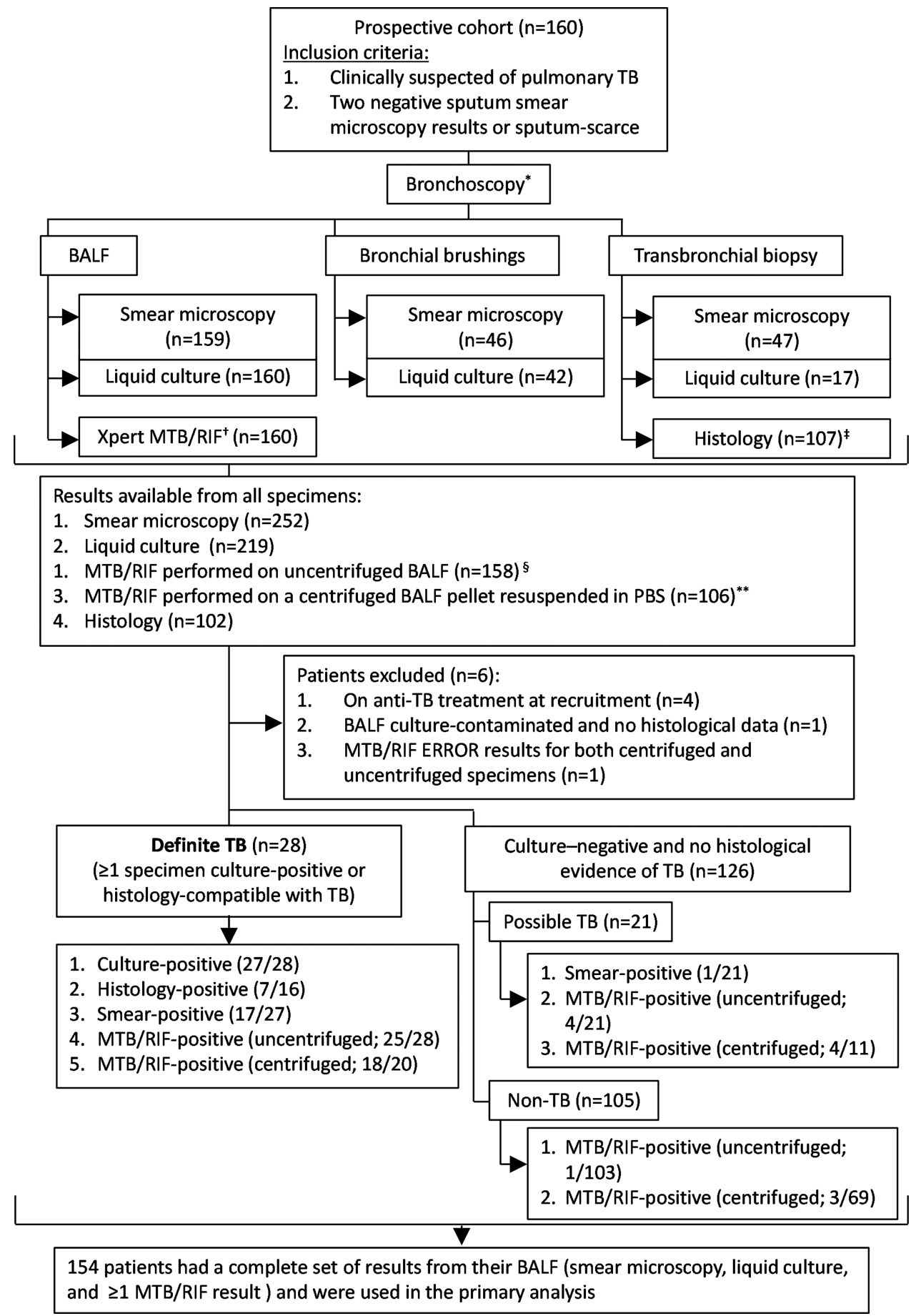

Figure 1 Study flow diagram showing the patients included in the analysis and test results.

*All patients provided bronchoalveolar lavage fluid. Bronchial brushings and biopsies were provided at the discretion of the attending clinician to the reference laboratory. Smear and culture were performed on these latter two specimens when possible by the reference laboratory.

TMTB/RIF was performed on fresh BALF from 82 patients. Fluid thawed from a frozen specimen was used for MTB/RIF for 78 patients recruited prior to the World Health Organization's endorsement of MTB/RIF.

¥Histology was considered compatible with active TB if necrotising or caseating granulomatous inflammation were observed by the reference laboratory. Five individuals had a record of histology being requested, but the result was missing.

$\S$ ne MTB/RIF ERROR result was recorded.

${ }^{* *}$ One MTB/RIF ERROR result and one INVALID result were recorded.

BALF, bronchoalveolar lavage fluid; PBS, phosphate buffer saline.

9/39 (23\%) and 4/15 (26\%) of patients were positive, respectively. All culture-positive bronchial brushings and/or transbronchial biopsies also had culture-positive BALF. Histological examination of transbronchial biopsies showed compatibility with active TB in 7 of the 102 cases $(7 \%)$ examined, with only one having culture- negative BALF. A greater proportion of culture-negative cases had a history of previous TB treatment compared with those with definite TB (39\% vs 18\%; $\mathrm{p}=0.031)$. The clinical, demographic and diagnostic characteristics of patients were similar across both phases (see online supplementary table S1). 
Table 1 Demographic and clinical characteristics

\begin{tabular}{|c|c|c|c|c|}
\hline & Cohort $(n=154)$ & $\begin{array}{l}\text { BALF culture positive } \\
\text { and/or histology compatible } \\
\text { with active TB }(n=28)\end{array}$ & $\begin{array}{l}\text { BALF culture negative } \\
\text { and no histological } \\
\text { evidence of active TB }(n=126)\end{array}$ & p Value \\
\hline Median age in years (IQR) & $46.1(33.1-55.7)$ & $42.5(30.1-54.3)$ & $47.5(33.9-57.1)$ & 0.318 \\
\hline Male gender (\%) & $83(54)$ & $11(39)$ & $72(57)$ & 0.093 \\
\hline Sputum-scarce (\%) & $111(72)$ & $20(71)$ & $91(72)$ & 0.918 \\
\hline HIV-infected* $\left.{ }^{*} \%\right)$ & $46(35)$ & $8(40)$ & $38(35)$ & 0.660 \\
\hline Median CD4 count (cells/ $\mu$ I) (IQR) if HIV infected $t$ & $165(67-406)$ & $137(55-645)$ & $179(67-406)$ & 0.783 \\
\hline Previous TB treatment $\ddagger(\%)$ & $52(35)$ & $5(18)$ & 47 (39) & 0.035 \\
\hline Smoker§ (past or current) (\%) & $42(30)$ & $5(21)$ & $37(32)$ & 0.146 \\
\hline
\end{tabular}

\section{Diagnostic accuracy of MTB/RIF performed on uncentrifuged BALF}

Of the 27 culture-positive patients, 25 had MTB/RIF-positive uncentrifuged BALF. The sensitivity of MTB/RIF was $93 \%$ $(25 / 27 ; 95 \%$ CI $77 \%$ to $98 \%)$ and significantly higher than smear microscopy performed on the same fluid, which had a sensitivity of $58 \%(15 / 26 ; 39 \%$ to $75 \%$; $<<0001$; table 2$)$. Five of 127 culture-negative cases had MTB/RIF-positive uncentrifuged BALF, resulting in a specificity of $96 \%$ (91\% to $98 \%)$, which was similar to smear microscopy $(99 \%(126 / 127 ; 96 \%$ to $100 \%) ; \mathrm{p}=0.121)$. The positive predictive value (PPV) and negative predictive value (NPV) of MTB/RIF were $83 \%(25 / 30$; $67 \%$ to $93 \%)$ and $98 \%(120 / 122 ; 94 \%$ to $100 \%)$, respectively. HIV coinfection did not impact sensitivity, specificity (table 2) or liquid culture time to positivity (TTP). When MTB/RIF accuracy was evaluated using a composite reference standard (culture positivity from any specimen and/or histological evidence of active TB from a transbronchial biopsy), the sensitivity and specificity were similar to those obtained in the primary analysis using either definite TB versus possible and non-TB combined (see online supplementary table S2); definite and possible TB combined versus non-TB (see online supplementary table S3); or definite $\mathrm{TB}$ versus non-TB with the possible $\mathrm{TB}$ group excluded (see online supplementary table S4).

\section{Effect of BALF centrifugation on MTB/RIF accuracy}

Of the 100 patients with BALF available for centrifugation, 19 were culture positive for TB. Centrifugation did not improve sensitivity and high specificity was retained (table 2), and did not affect $\mathrm{C}_{\mathrm{T}}$ values (see online supplementary material).

\section{Analysis of patients who were MTB/RIF positive with no culture or histological evidence of active TB}

We detected $12 \mathrm{MTB} / \mathrm{RIF}$-positive, culture-negative results (from 11 patients). Five of the eleven patients had sufficient BALF stored for a Genotype MTBDRplus assay, the specimens of which were all detected as containing $M$ tuberculosis complex DNA. When these five patients were added to the definite TB group, the sensitivity and specificity of an uncentrifuged MTB/RIF changed from 92.6\% (25/27) and 96\% (120/ $125)$ to $94 \%(30 / 32)$ and $100 \%(120 / 120)$, respectively. Further clinical information about the patients who were MTB/RIF positive, culture negative is given in the online supplementary material.

\section{Relationship of MTB/RIF-generated cycle threshold values} with bacterial load

MTB/RIF-generated $C_{T}$ values had a strong correlation with smear grade $(p=0.008)$ and liquid culture TTP $(p=0.001$; $\mathrm{R}^{2}=0.49$ ) (figure 2).

\section{Accuracy of MTB/RIF for the detection of resistance to rifampicin}

BALF from four patients was detected as resistant to rifampicin. One was culture positive and phenotypically resistant to rifampicin and isoniazid. The remaining three were culture negative and did not have phenotypic drug susceptibility data. Of these, one had sufficient BALF fluid remaining for a Genotype MTBDRplus assay, and was $M$ tuberculosis complex positive but rifampicin and isoniazid susceptible. Of the three, two completed standard first-line treatment and one was not placed on treatment. All had symptoms resolved at follow-up.

\section{Impact of MTB/RIF on time to TB detection (phase one vs phase two)}

The time-point-specific proportions of definite TB cases detected by MTB/RIF (uncentrifuged and centrifuged), smear microscopy (on any specimen), histology and liquid culture are shown in figure 3 for phase two when MTB/RIF was used for patient management. Eighteen definite TB cases were detected. The median time to detection (IQR) for MTB/RIF was 0 days $(0-0)$, compared with 0 days $(0-1)$ for smear microscopy, 3 days (1-10) for histology and 15 days (11-20) for liquid culture ( $p$ values of $0.229,0.001$ and $<0.001$, respectively compared with MTB/RIF). The median (IQR) time to detection for smearnegative TB shortened from 29 days $(18-41)$ to 0 days $(0-0$; $\mathrm{p}<0.001$ ) (all smear-negative TB cases were detected by MTB/ RIF in phase two) (table 3 ).

\section{Impact of Xpert MTB/RIF on the proportion of patients} initiating anti-TB treatment (phase one vs phase two) Table 3 and figure 4 summarise differences in treatment initiation between the two phases. Overall, a similar proportion of patients were initiated on anti-TB treatment when phase one was compared with phase two $(26 \%(17 / 67)$ vs $36 \%(26 / 73)$; $\mathrm{p}=0.196)$. Of those initiated onto treatment, a similar proportion were confirmed TB cases $(41 \%$ (7/17) for phase one vs $68 \%(15 / 23)$ for phase two; $p=0.150)$. The proportion of patients initiating treatment in each phase based on the results of smear microscopy, histology, MTB/RIF, culture or clinical and 
Table 2 Accuracy of Xpert MTB/RIF for the detection of culture-positive TB in bronchoalveolar lavage fluid

\begin{tabular}{|c|c|c|c|c|c|c|}
\hline & \multicolumn{2}{|l|}{$\begin{array}{l}\text { All patients } \\
\mathrm{n}=154\end{array}$} & \multicolumn{2}{|l|}{$\begin{array}{l}\text { HIV uninfected } \\
\mathrm{n}=84\end{array}$} & \multicolumn{2}{|l|}{$\begin{array}{l}\text { HIV infected } \\
n=46\end{array}$} \\
\hline & $\begin{array}{l}\text { Sensitivity, \% } \\
(95 \% \text { Cl) }\end{array}$ & $\begin{array}{l}\text { Specificity, \% } \\
(95 \% \text { Cl) }\end{array}$ & $\begin{array}{l}\text { Sensitivity, \% } \\
(95 \% \text { Cl) }\end{array}$ & $\begin{array}{l}\text { Specificity, \% } \\
(95 \% \text { Cl) }\end{array}$ & $\begin{array}{l}\text { Sensitivity, \% } \\
(95 \% \text { Cl) }\end{array}$ & $\begin{array}{l}\text { Specificity, \% } \\
(95 \% \text { Cl) }\end{array}$ \\
\hline Smear microscopy & $\begin{array}{l}57.7 \text { (39 to } 74.5) \\
15 / 26\end{array}$ & $\begin{array}{l}99.3(95.7 \text { to } 99.9) \\
126 / 127\end{array}$ & $\begin{array}{l}58.4 \text { (32 to } 80.7) \\
7 / 12\end{array}$ & $\begin{array}{l}100(95 \text { to } 100) \\
72 / 72\end{array}$ & $\begin{array}{l}57.2(25.1 \text { to } 84.2) \\
4 / 7\end{array}$ & $\begin{array}{l}97.4 \text { (86.6 to } 99.6) \\
37 / 38\end{array}$ \\
\hline MTB/RIF performed on uncentrifuged BALF & $\begin{array}{l}92.6(76.7 \text { to } 98) \\
25 / 27 \\
(p=0.001)\end{array}$ & $\begin{array}{l}96(91 \text { to } 98.3) \\
120 / 125\end{array}$ & $\begin{array}{l}100(75.8 \text { to } 100) 12 / 12 \\
(\mathrm{p}=0.02)^{*}\end{array}$ & $\begin{array}{l}95.9(88.5 \text { to } 98.6) \\
69 / 72\end{array}$ & $\begin{array}{l}75 \text { (41 to } 92.9) \\
6 / 8\end{array}$ & $\begin{array}{l}94.5(81.9 \text { to } 98.5) \\
34 / 36\end{array}$ \\
\hline MTB/RIF performed on uncentrifuged BALF in patients who were smear negative & $\begin{array}{l}81.9 \text { (52.4 to } 94.9) \\
9 / 11\end{array}$ & $\begin{array}{l}96.8 \text { (92 to } 98.8) \\
120 / 124\end{array}$ & $\begin{array}{l}100(56.6 \text { to } 100) \\
5 / 5\end{array}$ & $\begin{array}{l}95.9(88.5 \text { to } 98.6) \\
69 / 72\end{array}$ & $\begin{array}{l}33.4(6.2 \text { to } 79.3) \\
1 / 3\end{array}$ & $\begin{array}{l}97.2(85.5 \text { to } 99.5) \\
34 / 35\end{array}$ \\
\hline MTB/RIF performed on a resuspended pellet of centrifuged BALF & $\begin{array}{l}94.8(75.4 \text { to } 99.1) \\
18 / 19\end{array}$ & $\begin{array}{l}91.4(83.3 \text { to } 95.8) \\
74 / 81\end{array}$ & $\begin{array}{l}88.9(56.6 \text { to } 98.1) \\
8 / 9\end{array}$ & $\begin{array}{l}95.3 \\
(84.3 \text { to } 98.7) \\
40 / 42\end{array}$ & $\begin{array}{l}100 \\
(43.9 \text { to } 100) \\
3 / 3\end{array}$ & $\begin{array}{l}87 \\
(67.9 \text { to } 95.5) \\
20 / 23\end{array}$ \\
\hline \multirow[t]{2}{*}{ Two MTB/RIF assays (performed on uncentrifuged and centrifuged BALF, respectively) } & $\begin{array}{l}100(83.2 \text { to } 100) \\
19 / 19\end{array}$ & $\begin{array}{l}89.9(81.3 \text { to } 94.8) \\
71 / 79\end{array}$ & $\begin{array}{l}100(70.1 \text { to } 100) \\
9 / 9\end{array}$ & $\begin{array}{l}92.9(81 \text { to } 97.6) \\
39 / 42\end{array}$ & $\begin{array}{l}100(43.9 \text { to } 100) \\
3 / 3\end{array}$ & $\begin{array}{l}85.8(65.4 \text { to } 95.1) \\
18 / 21\end{array}$ \\
\hline & $\begin{array}{l}\text { PPV† } \\
(95 \% \mathrm{Cl})\end{array}$ & $\begin{array}{l}\text { NPV† } \\
(95 \% \mathrm{Cl})\end{array}$ & $\begin{array}{l}\text { PPV† } \\
(95 \% \mathrm{Cl})\end{array}$ & $\begin{array}{l}\mathrm{NPV \dagger} \\
(95 \% \mathrm{Cl})\end{array}$ & $\begin{array}{l}\mathrm{PPV} \dagger \\
(95 \% \mathrm{Cl})\end{array}$ & $\begin{array}{l}\mathrm{NPV \dagger} \\
(95 \% \mathrm{Cl})\end{array}$ \\
\hline Smear microscopy & $\begin{array}{l}93.8(71.7 \text { to } 98.9) \\
15 / 16\end{array}$ & $\begin{array}{l}92(86.2 \text { to } 95.5) \\
126 / 137\end{array}$ & $\begin{array}{l}100(64.6 \text { to } 100) \\
7 / 7\end{array}$ & $\begin{array}{l}93.6(85.7 \text { to } 97.2) \\
72 / 77\end{array}$ & $\begin{array}{l}80 \text { (37.6 to } 96.4) \\
4 / 5\end{array}$ & $\begin{array}{l}92.5(80.2 \text { to } 97.5) \\
37 / 40\end{array}$ \\
\hline MTB/RIF performed on uncentrifuged BALF & $\begin{array}{l}83.4(66.5 \text { to } 92.7) \\
25 / 30\end{array}$ & $\begin{array}{l}98.4(94.3 \text { to } 99.6) \\
120 / 122\end{array}$ & $\begin{array}{l}80(54.9 \text { to } 93) \\
12 / 15\end{array}$ & $\begin{array}{l}100(94.8 \text { to } 100) \\
69 / 69\end{array}$ & $\begin{array}{l}75(41 \text { to } 92.9) \\
6 / 8\end{array}$ & $\begin{array}{l}94.5(81.9 \text { to } 98.5) \\
34 / 36\end{array}$ \\
\hline MTB/RIF performed on uncentrifuged BALF in patients who were smear negative & $\begin{array}{l}69.3(42.4 \text { to } 87.4) \\
9 / 13\end{array}$ & $\begin{array}{l}98.4(94.3 \text { to } 99.6) \\
120 / 122\end{array}$ & $\begin{array}{l}62.5 \text { (30.6 to } 86.4) \\
5 / 8\end{array}$ & $\begin{array}{l}100(94.8 \text { to } 100) \\
69 / 69\end{array}$ & $\begin{array}{l}50(9.5 \text { to } 90.6) \\
1 / 2\end{array}$ & $\begin{array}{l}94.5 \text { (81.9 to } 98.5) \\
34 / 36\end{array}$ \\
\hline MTB/RIF performed on a resuspended pellet of centrifuged BALF & $\begin{array}{l}72(52.5 \text { to } 85.8) \\
18 / 25\end{array}$ & $\begin{array}{l}98.7 \% \text { (92.9 to } 99.8) \\
74 / 75\end{array}$ & $\begin{array}{l}80(49.1 \text { to } 94.4) \\
8 / 10\end{array}$ & $\begin{array}{l}97.6(87.5 \text { to } 99.6) \\
40 / 41\end{array}$ & $\begin{array}{l}50(18.8 \text { to } 81.3) \\
3 / 6\end{array}$ & $\begin{array}{l}100(83.9 \text { to } 100) \\
20 / 20\end{array}$ \\
\hline Two MTB/RIF assays (performed on uncentrifuged and centrifuged BALF, respectively) & $\begin{array}{l}70.4 \text { (51.6 to } 84.2) \\
19 / 27\end{array}$ & $\begin{array}{l}100 \\
(94.9 \text { to } 100) \\
71 / 71\end{array}$ & $\begin{array}{l}75 \\
(46.8 \text { to } 91.2) \\
9 / 12\end{array}$ & $\begin{array}{l}100 \\
(91.1 \text { to } 100) \\
39 / 39\end{array}$ & $\begin{array}{l}50 \\
(18.8 \text { to } 81.3) \\
3 / 6\end{array}$ & $\begin{array}{l}100 \\
(82.5 \text { to } 100) \\
18 / 18\end{array}$ \\
\hline
\end{tabular}

†Based on TB prevalences of $18 \%, 14 \%$ and $18 \%$ overall and in the patient groups with HIV infection and without HIV infection.

BALF, bronchoalveolar lavage fluid; NPV, negative predictive value; PPV, predictive value; TB, tuberculosis. 
Figure 2 Relationship between MTB/ RIF-generated cycle threshold $\left(C_{T}\right)$ values and bacterial load in BALF.
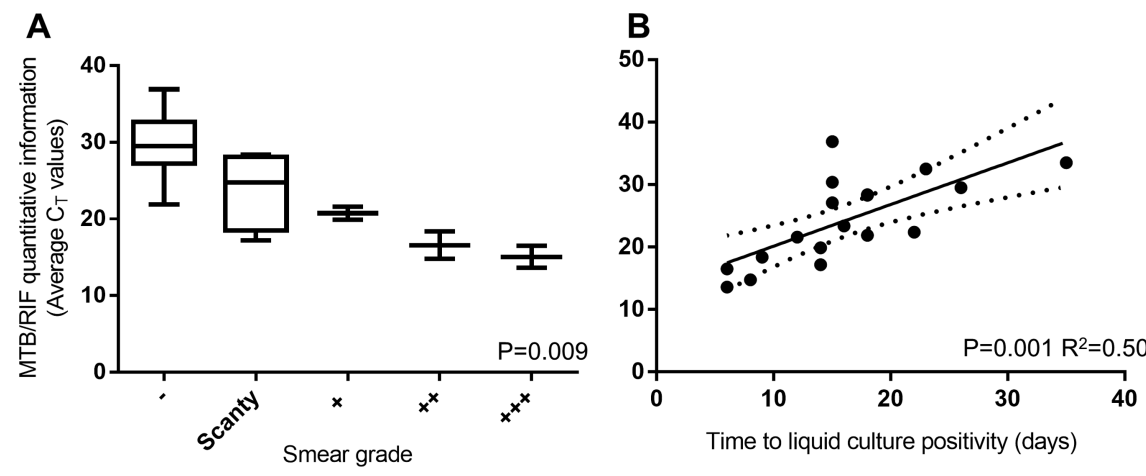

radiological findings alone (empirical treatment) is shown in figure $4 \mathrm{~A}$ and $\mathrm{B}$. In phase two, a smaller proportion of the patients were initiated empirically $(79 \%(11 / 14)$ vs $28 \%$ (10/ $25) ; \mathrm{p}=0.026)$ and the proportion of patients who initiated treatment on the basis of a positive rapid test result (smear microscopy in phase one with the addition of $\mathrm{MTB} / \mathrm{RIF}$ in phase two) increased from $14 \%(2 / 14)$ to $80 \%(20 / 25$; $\mathrm{p}<0.001)$.

Of those treated on empirical grounds, 25\% (7/17) and 58\% $(15 / 26)$ were later shown to be definite TB from a specimen collected at recruitment in phase one and phase two $(p=0.312)$. The median time to empirical TB treatment initiation did not differ in phase one versus phase two (2 (1-24) days vs 40 (1079) days; $p=0.071)$.

\section{Impact of Xpert MTB/RIF on the time to anti-TB treatment initiation (phase one vs phase two)}

The median (IQR) times to anti-TB treatment initiation for phase one and phase two when MTB/RIF was used for patient management were $10(1-49)$ days and $7(0-21)$ days, respectively $(p=0.330)$ (figure 5$)$. For patients with definite TB, these times were $5(1-57)$ and $2(0-7)$ days, respectively $(p=0.358)$. For smear-negative patients, the median times to treatment initiation across each phase were 15 (1-50) days and $7(0-33)$ days, respectively $(p=0.55)$. When the time-specific proportion of patients initiating anti-TB treatment was compared at time points of $2,10,20,30,40$ and 50 days, there were no differences detected.

\section{DISCUSSION}

Information regarding the accuracy and impact of MTB/RIF when performed on BALF is limited. The effect of HIV infection, specimen concentration and impact on time to TB diagnosis and treatment initiation is unknown. Bronchoscopy is used frequently, when appropriate, in major cities in South Africa. In the Western Cape Province there are around eight hospitals (public and private facilities) with bronchoscopy facilities. In public healthcare facilities in Cape Town around 600 bronchoscopies are performed each year. The key findings of our study are as follows: MTB/RIF significantly outperformed smear microscopy on BALF, improved the time to diagnosis and detected $\geq 80 \%$ of TB cases that were smear negative; centrifugation did not improve accuracy; empirical treatment was higher with smear microscopy as the only available rapid test; and hence MTB/RIF did not significantly impact the overall proportion of patients initiating treatment. Our study highlights the possibility that the impact of MTB/RIF may be overestimated due to high rates of empirical treatment when smear microscopy is the only available rapid test.

Four studies examined MTB/RIF performance in BALF $^{11} 122021$ and all possessed a small number of specimens, were conducted retrospectively on biobanked specimens, and had less than five TB cases. In our study of 154 patients, the sensitivity of MTB/RIF on BALF was $94 \%$, similar to other respiratory specimens, ${ }^{10-12} 2021$ and exceeded that reported for other commercially available nucleic acid amplification tests. ${ }^{22-24} \mathrm{We}$ detected 11 patients who were MTB/RIF positive and culture

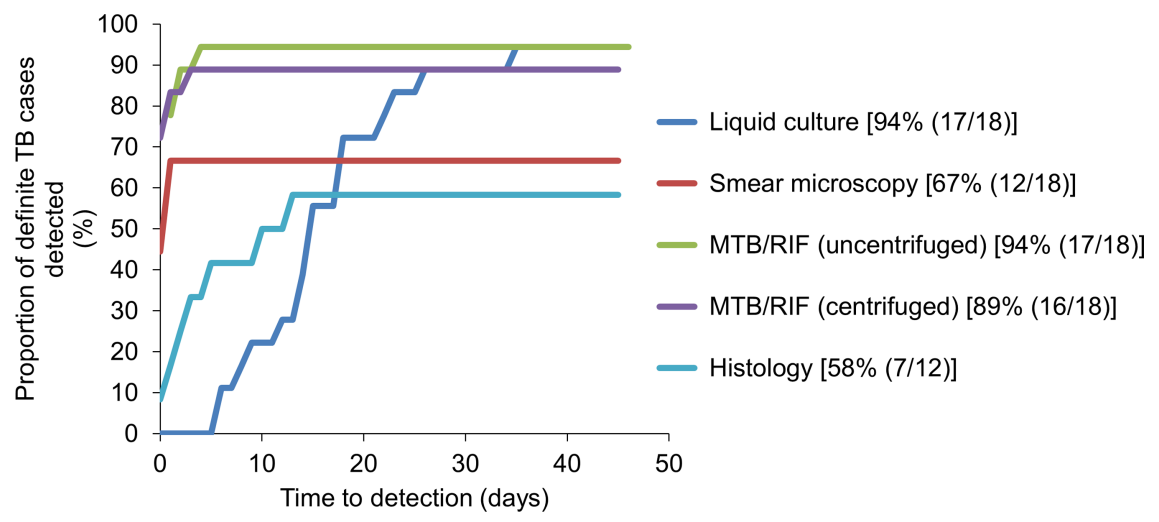

Figure 3 Time-specific proportion of definite TB cases detected by different diagnostic methods when MTB/RIF results were used for patient management in phase two.

*Positive results are included here from different specimens for smear microscopy: of all 12 smear-positives, two did not have smear-positive bronchoalveolar lavage fluid but did have smear-positive bronchial biopsies. Histology was only performed on bronchial biopsies in 12 of the 18 definite TB cases. For liquid culture, all 17 detected individuals had culture-positive bronchoalveolar lavage fluid and there were no additional cases detected on bronchial brushings and biopsies. MTB/RIF was performed only on bronchoalveolar lavage fluid. 
Table 3 Impact of MTB/RIF on the times to TB detection and initiation of anti-TB treatment before (phase one) and after (phase two) it was used for patient management

\begin{tabular}{|c|c|c|c|}
\hline Outcome & Phase one & Phase two & p Value \\
\hline \multicolumn{4}{|l|}{ Days-to-result (IQR) } \\
\hline Definite TB & $6(1-29)$ & $0(0-1)$ & $<0.001$ \\
\hline Smear-negative TB & $29(18-41)$ & $0(0-0)$ & $<0.001$ \\
\hline Proportion on treatment (positive over total; \%) & $26(17 / 67)$ & $36(26 / 73)$ & 0.196 \\
\hline Proportion who initiated treatment empirically* & $79(11 / 14)$ & $28(10 / 25)$ & 0.026 \\
\hline \multicolumn{4}{|c|}{ Proportion of all patients in each phase who initiated treatment according to final microbiological diagnosis (positive over total; \%) } \\
\hline Definite TB & $41(7 / 17)$ & $68(15 / 23)$ & 0.150 \\
\hline Possible-TB & $59(10 / 17)$ & $42(11 / 26)$ & 0.313 \\
\hline \multicolumn{4}{|l|}{ Days to treatment initiation (IQR) } \\
\hline All patients & $10(1-49)$ & $4(0-16)$ & 0.330 \\
\hline Definite TB & $5(1-57)$ & $2(0-7)$ & 0.358 \\
\hline
\end{tabular}

negative. We have previously demonstrated that such patients likely represent true TB cases. ${ }^{25}$

Similar to what has previously been documented in sputum, ${ }^{25} 26$ we found that MTB/RIF-generated quantitative information strongly correlated with markers of bacterial load in BALF, such as smear grade and liquid culture TTP. This has implications for the use of $\mathrm{MTB} / \mathrm{RIF} \mathrm{C}_{\mathrm{T}}$ values to assess disease severity, ${ }^{27}$ identify those at risk of treatment failure ${ }^{28-30}$ and stratify patients likely to be highly infectious. ${ }^{31}$

Previously we demonstrated that the use of $5-10 \mathrm{ml}$ of centrifuged urine from HIV-infected sputum-scarce individuals improved MTB/RIF performance compared with uncentrifuged
Figure 4 Venn diagrams (A) and bar graph (B) showing the proportion of patients either initiated onto treatment on the basis of different positive test results, or who treated empirically in the absence of a positive result before (phase one) and after (phase two) Xpert MTB/RIF was used for patient management.

* $22 \%$ of 64 patients in phase one and $35 \%$ of patients in phase two were placed on treatment and have known dates of treatment initiation. Three patients in phase one and one patient in phase two who were placed on antiTB treatment were missing the date of anti-TB treatment initiation and are omitted. Histology detected no patients in phase one and seven patients in phase two (all of which were initiated onto treatment before the histology result was available as they were also smear- or MTB/RIFpositive).

tEmpiric anti-TB treatment is defined as the initiation of treatment on clinical grounds in the absence of positive smear microscopy, histology or culture result (in phase one), plus the absence of a positive MTB/RIF result (phase two only), at the time of treatment initiation.
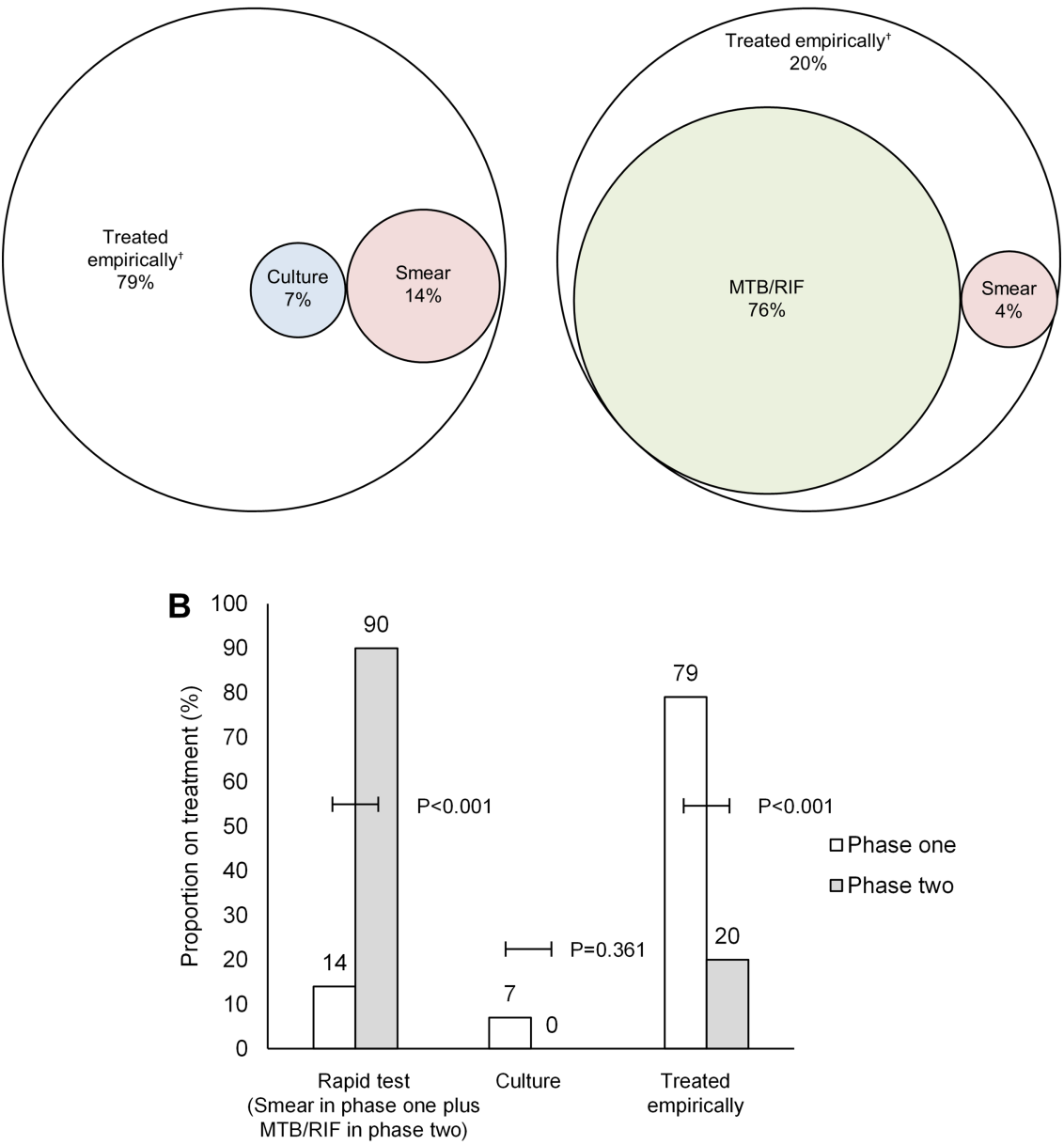

$\square$ Phase one

$\square$ Phase two 


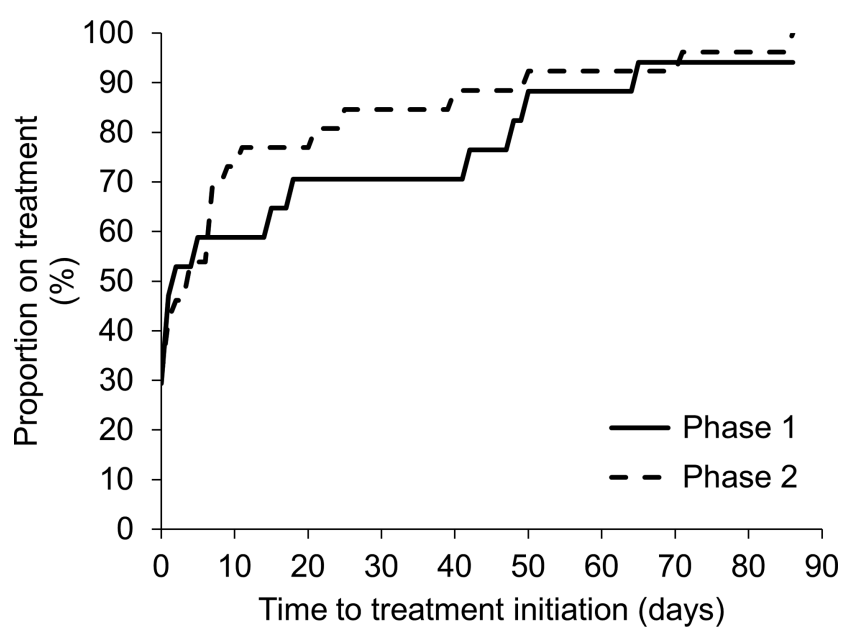

Figure 5 Time-specific proportion of patients initiating anti-TB treatment before (phase one) and after (phase two) MTB/RIF was used for patient management.

urine. ${ }^{32}$ We did not find a significant improvement in sensitivity when a similar volume of BALF was centrifuged. This is likely because the concentration of bacilli in fluid sampled directly from the site of disease (the lung) is above the limit of detection of the assay without the need for centrifugation. One millilitre of uncentrifuged BALF is sufficient for accurate performance of the MTB/RIF assay.

Only two studies have, to the best of our knowledge, examined the impact of MTB/RIF on time to TB diagnosis and TB treatment initiation ${ }^{33}{ }^{34}$; however, they were performed on sputum. We found MTB/RIF to provide a result in significantly more patients than smear microscopy, and within a similar timeframe (0-1 days), thereby significantly reducing the time to diagnosis in patients with smear-negative BALF.

Although MTB/RIF implementation facilitated a more rapid diagnosis of $\mathrm{TB}$, it did not result in detectable improvements in the time to TB treatment initiation. This is because our study was underpowered to detect small differences in this outcome (sample size calculations suggest that we had $77 \%$ power to detect a $20 \%$ difference in the proportion initiating treatment between study phases and only a $30 \%$ power to detect a difference of 3 days in the time to treatment initiation). This arose due to MTB/RIF becoming the standard care at the study site following endorsement of the test by $\mathrm{WHO}^{7}$ and the South African National Department of Health, which resulted in us being ethically obligated to end phase one and use MTB/RIF for patient management. It appears that the potential impact of MTB/RIF on improving time to treatment initiation in phase two was mitigated by high rates of rapidly initiated empirical anti-TB treatment in the absence of a positive microbiological result in phase one. A study assessing the impact of sputum-based MTB/RIF among hospitalised Ugandan inpatients has reported a similar phenomenon. ${ }^{34}$ These findings underscore the importance of assessing the impact of diagnostic tests in a routine environment, where clinical practice has evolved to compensate for the poor performance of smear microscopy. Large multicentre studies are required to definitively assess this question.

This study has limitations. Although its use is increasing, bronchoscopy is not yet widely available in resource-poor settings and is usually only at a tertiary level where the burden of HIV (and smear-negative or sputum-scarce TB) is highest. Our data will also have relevance to low-burden settings where there are large immigrant populations, and where bronchoscopy is widely available and used. We did not examine the utility of $\mathrm{MTB} / \mathrm{RIF}$ on induced sputum, which may reduce the need for MTB/RIF testing on BALF from patients with suspected TB. Additionally, for the secondary analysis, not all patients had test results for each of the three biological specimens examined, and this may have introduced sampling bias. We may have used a suboptimal volume of BALF for centrifugation; however, we were limited by available volumes after specimens were sent for routine laboratory testing. The use of frozen specimens from some patients may have impacted MTB/RIF accuracy, though published data suggest that the effect, if any, is likely to be minimal. ${ }^{9} 2535$

In summary, $\mathrm{MTB} / \mathrm{RIF}$ on the BALF of patients who are smear negative or sputum scarce has shown excellent performance for the detection of $\mathrm{TB}$ in a HIV-prevalent setting. Although in patients with smear-negative BALF the time to TB result significantly improved, this did not translate into a higher proportion of patients initiating treatment. However, it did reduce the proportion of patients initiating treatment on empirical grounds. The high baseline levels of empirical treatment in our setting may undermine the potential impact of MTB/RIF, but this requires prospective investigation.

Acknowledgements The authors are indebted to the patients who participated in this study, as well as Sister Marietjie Pretorius, Ms Dolphina Cogill and Ms Vonnita Louw for excellent support.

Contributors Conception and design: GT, JP, HK, KD. Specimen collection and clinical input: $K D, G C, G S, R M, P G, B M, B A$ and LL. Analysis and interpretation: GT, $J P, K D, G C, M S$ and UG. Drafting the manuscript for important intellectual content: $\mathrm{GT}$, JP, GC, MS and KD.

Funding This work was supported by an EU-FP7 award (TBSusgent F3-2008223340). GT, JP and UG are supported by the EDCTP (TB-NEAT IP.09.32040.009), the South African National Research Foundation (GT, UG), the Claude Leon Foundation (GT) and a Wellcome Trust Training Fellowship (GT). JP is supported by a Discovery Foundation Fellowship, the Fogarty International Clinical Research Scholars/Fellows Support Centre National Institutes of Health grant R24TW007988 and SATBAT (JP). KD is supported by the EDCTP (TB-NEAT, TESA) and the SA DST and NRF (SARChl).

\section{Competing interests None.}

Patient consent Patients provided written informed consent for study participation.

Ethics approval The study protocol was approved by the University of Cape Town Faculty of Health Sciences Research Ethics Committee (\#421/2006).

Provenance and peer review Not commissioned; externally peer reviewed.

\section{REFERENCES}

1 Corbett EL, Watt CJ, Walker N, et al. The growing burden of tuberculosis: global trends and interactions with the HIV epidemic. Arch Intern Med 2003;163:1009.

2 Getahun $\mathrm{H}$, Harrington $\mathrm{M}, \mathrm{O}^{\prime}$ Brien $\mathrm{R}$, et al. Diagnosis of smear-negative pulmonary tuberculosis in people with HIV infection or AIDS in resource-constrained settings: informing urgent policy changes. Lancet 2007;369:2042-49.

3 Peter JG, Theron G, van Zyl-Smit R, et al. Diagnostic accuracy of a urine LAM strip-test for TB detection in HIV-infected hospitalised patients. Eur Respir J 2012:40:1211-20.

4 WHO. Global tuberculosis control 2011. Publication no. WHO/HTM/TB/2011.16 Geneva, Switzerland: World Health Organization, 2011.

5 Dheda K, Lampe FC, Johnson MA, et al. Outcome of HIV-associated tuberculosis in the era of highly active antiretroviral therapy. J Infect Dis 2004;190:1670.

6 Hepple P, Ford N, McNerney R. Microscopy compared to culture for the diagnosis of tuberculosis in induced sputum samples: a systematic review. Int I Tuberc Lung Dis 2012;16:579.

7 WHO. Automated real-time nucleic acid amplification technology for rapid and simultaneous detection of tuberculosis and rifampicin resistance: Xpert MTB/RIF SYSTEM. Publication number WHO/HTM/TB/2011.4. Geneva, Switzerland: World Health Organization, 2011.

8 Boehme CC, Nabeta P, Hillemann D, et al. Rapid molecular detection of tuberculosis and rifampin resistance. N Engl J Med 2010;363:1005-15.

9 Steingart K, Sohn H, Schiller I, et al. Xpert ${ }^{\circledR}$ MTB/RIF assay for pulmonary tuberculosis and rifampicin resistance in adults. Cochrane Database Syst Rev 2013; (1):CD009593. 
10 Chang K, Lu W, Wang J, et al. Rapid and effective diagnosis of tuberculosis and rifampicin resistance with Xpert MTB/RIF assay: a meta-analysis. J Infect 2012;64:580-8.

11 Teo J, Jureen R, Chiang D, et al. Comparison of two nucleic acid amplification assays, the Xpert MTB/RIF assay and the amplified Mycobacterium tuberculosis Direct assay, for detection of Mycobacterium tuberculosis in respiratory and nonrespiratory specimens. J Clin Microbiol 2011;49:3659-62.

12 Armand S, Vanhuls P, Delcroix G, et al. Comparison of the Xpert MTB/RIF test with an IS6110-TaqMan real-time PCR assay for direct detection of Mycobacterium tuberculosis in respiratory and nonrespiratory specimens. J Clin Microbiol 2011;49:1772-76.

13 Cruciani M, Scarparo C, Malena M. Meta-analysis of BACTEC MGIT 960 and BACTEC 460 TB, with or without solid media, for detection of mycobacteria. J Clin Microbiol 2004;42:2321-25.

14 Scarparo C, Ricordi P, Ruggiero G, et al. Evaluation of the fully automated BACTEC MGIT 960 system for testing susceptibility of Mycobacterium tuberculosis to pyrazinamide, streptomycin, isoniazid, rifampin, and ethambutol and comparison with the radiometric BACTEC 460TB method. I Clin Microbiol 2004;42:1109.

15 Helb D, Jones M, Story E, et al. Rapid detection of Mycobacterium tuberculosis and rifampin resistance by use of on-demand, near-patient technology. I Clin Microbiol 2010;48:229-37.

16 Xpert MTB/RIF [package insert]. Cepheid S, CA, 300-7810 Rev. A, April 2009.

17 Barnard M, Gey van Pittius NC, van Helden P, et al. Diagnostic performance of Genotype ${ }^{\circledR}$ MTBDRplus Version 2 line probe assay is equivalent to the Xpert $\circledast$ MTB/RIF assay. I Clin Microbiol 2012;50:2712-16.

18 Bossuyt PM, Reitsma JB, Bruns DE, et al. The STARD statement for reporting studies of diagnostic accuracy: explanation and elaboration. The Standards for Reporting of Diagnostic Accuracy Group. Croat Med J 2003;44:639-50.

19 Dean AGSK, Soe MM. OpenEpi: Open Source Epidemiologic Statistics for Public Health, Version 2.3.1. http://www.OpenEpi.com (updated 19 Sep 2010; accessed 30 Nov 2010).

20 Miller MB, Popowitch EB, Backlund MG, et al. Performance of Xpert MTB/RIF RUO assay and IS6110 real-time PCR for Mycobacterium tuberculosis detection in clinical samples. J Clin Microbiol 2011;49:3458.

21 Zeka AN, Tasbakan S, Cavusoglu C. Evaluation of the GeneXpert MTB/RIF assay for rapid diagnosis of tuberculosis and detection of rifampin resistance in pulmonary and extrapulmonary specimens. J Clin Microbiol 2011:49:4138-41.

22 Simonnet $\mathrm{C}$, Lacoste $\mathrm{V}$, Drogoul AS, et al. Automated extraction and amplification for direct detection of mycobacterium tuberculosis complex in various clinical samples. J Clin Microbiol 2011;49:1700-1.
23 Davis JL, Huang L, Worodria W, et al. Nucleic acid amplification tests for diagnosis of smear-negative TB in a high HIV-prevalence setting: a prospective cohort study. PLoS One 2011:6:e16321.

24 Chou PC, Wang CH, Huang CD, et al. Nucleic acid amplification test and bronchoscopy improve the diagnostic accuracy of smear-negative tuberculosis. Int J Tuberc Lung Dis 2012;16:1674-79.

25 Theron G, Peter J, van Zyl-Smit R, et al. Evaluation of the Xpert MTB/RIF assay for the diagnosis of pulmonary tuberculosis in a high HIV prevalence setting. Am J Respir Crit Care Med 2011;184:132-40.

26 Theron G, Pinto L, Peter J, et al. The use of an automated quantitative polymerase chain reaction (Xpert MTB/RIF) to predict the sputum smear status of tuberculosis patients. Clin Infect Dis 2012;54:384-88.

27 Perrin F, Woodward N, Phillips P, et al. Radiological cavitation, sputum mycobacterial load and treatment response in pulmonary tuberculosis. Int I Tuberc Lung Dis 2010;14:1596-602.

28 Hesseling A, Walzl G, Enarson D, et al. Baseline sputum time to detection predicts month two culture conversion and relapse in non-HIV-infected patients. Int I Tuberc Lung Dis 2010;14:560-70.

29 Visser ME, Stead MC, Walzl G, et al. Baseline predictors of sputum culture conversion in pulmonary tuberculosis: importance of cavities, smoking, time to detection and W-Beijing genotype. PLoS One 2012;7:e29588.

30 Bark CM, Thiel BA, Johnson JL. Pretreatment time to detection of mycobacterium tuberculosis in liquid culture is associated with relapse after therapy. J Clin Microbiol 2012;50:538-38.

31 Fennelly KP. An eXpert AFB smear? Clin Infect Dis 2012;54:389-91.

32 Peter JG, Theron G, Muchinga TE, et al. The diagnostic accuracy of urine-based Xpert MTB/RIF in HIV-infected hospitalized patients who are smear-negative or sputum scarce. PLoS One 2012;7:e39966.

33 Boehme CC, Nicol MP, Nabeta P, et al. Feasibility, diagnostic accuracy, and effectiveness of decentralised use of the Xpert MTB/RIF test for diagnosis of tuberculosis and multidrug resistance: a multicentre implementation study. The Lancet 2011:377:1495-505.

34 Yoon C, Cattamanchi A, Davis JL, et al. Impact of Xpert MTB/RIF testing on tuberculosis management and outcomes in hospitalized patients in Uganda. PLoS One 2012; 7 :e48599.

35 Moure R, Munoz L, Torres M, et al. Rapid detection of Mycobacterium tuberculosis complex and rifampin resistance in smear-negative clinical samples by use of an integrated real-time PCR method. J Clin Microbiol 2011;49:1137-39. 